\title{
Agilität und Agilitätsmanagement
}

\author{
Im Beitrag beschäftigen sich die Autoren mit einem der aktuellen Top- \\ Trends in der Managementliteratur und der betrieblichen Praxis - dem \\ Agilitätsmanagement. Nach einer kurzen Einführung wird skizziert, welche \\ Managementaufgaben durch Agilität angesprochen werden und wie die \\ Ambidextriedebatte hilft festzustellen, wann sich Agilität lohnt.
}

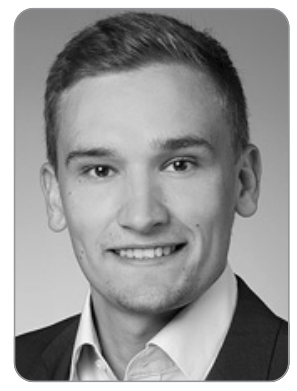

\section{Matthias Sellinger}

ist wissenschaftlicher Mitarbeiter an der Hochschule für Wirtschaft und Gesellschaft Ludwigshafen. Bevorzugte Forschungsgebiete: Innovationsmanagement, Neuproduktentwicklung, Individuelle und organisationale Adaptivität.

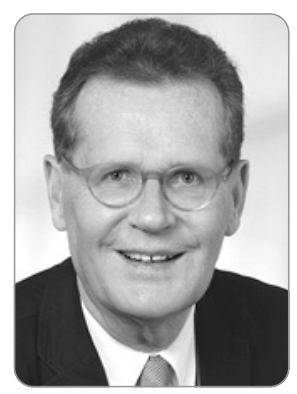

Prof. Dr. Dieter Thomaschewski ist Professor für allg. Betriebswirtschaftslehre insbesondere Management an der Hochschule für Wirtschaft und Gesellschaft Ludwigshafen. Bevorzugte Forschungsgebiete: Strategische Unternehmensführung, Internationales Management, Wachstumsstrategien.

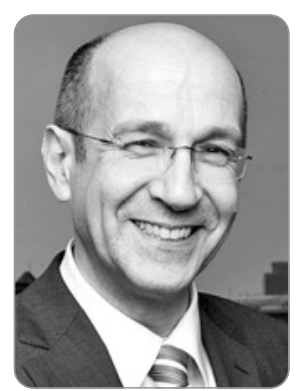

\section{Prof. Dr. Rainer Völker}

ist Professor für allg. Betriebswirtschaftslehre insbesondere Unternehmensführung an der Hochschule für Wirtschaft und Gesellschaft Ludwigshafen. Bevorzugte Forschungsgebiete: Innovationsmanagement, Nachhaltigkeitsmanagement, Strategisches Management.

Stichwörter: Agilität, Agilitätsmanagement, Ambidextrie, Agiles Management, Agile Organisation

\section{Begriff und Bedeutung}

"Agilität" ist eines der neuen Themen, welches es auf einen der Top-Plätze in der Managementliteratur, der Beratung, der Managementseminare und damit auch der betrieblichen Praxis geschafft hat. Die reine Wortbedeutung des Begriffes der Agilität beinhaltet zunächst Eigenschaften wie "Beweglichkeit” oder „Flexibilität”. Im betriebs- wirtschaftlichen Kontext findet sich der Begriff Agilität zum ersten Mal als Konzept in der Mitte der 80-er Jahre bei Moss-Kanter (1985) und Peters (1986). Diese beschrieben schon damals Agilität als Eigenschaft von Unternehmen, adaptiv mit Veränderungen im Marktumfeld umzugehen.

Insbesondere fand der Begriff jedoch Ende der 90-er Jahre durch den Einzug in den IT-Bereich das erste Mal große Bedeutung. Dies mündete in der Erstellung des agilen Manifests im Jahr 2001. Bestehend aus 4 Axiomen und 12 Prinzipien (vgl. Beck et al., 2001), läutete das Manifest eine rasante Entwicklung agiler Methoden, wie insbesondere Scrum und agiles Projektmanagement, ein. Mittlerweile werden entsprechende Methoden und agile Projektorganisationsformen auch im Nicht-IT Umfeld erfolgreich eingesetzt. So z.B. empfiehlt auch heute der Erfinder und Protagonist des State-Gate-Ansatzes - Robert G. Cooper - agile Vorgehensweisen in der Neuproduktentwicklung im Fertigungsbereich einzusetzen (vgl. Cooper, 2018, S. 15 ff.). Darüber hinaus werden Grundprinzipien der Agilität im Rahmen von Organisation und Führung zur Neugestaltung der Unternehmensführung herangezogen (vgl. Kalenda et al., 2018). Die Ziele bleiben ähnliche wie in der Softwareentwicklung: Man möchte eine höhere Reaktionsfähigkeit auf Veränderungen (Reagieren), eine verbesserte Fähigkeit zur Selbstveränderung (Lernen) und eine Stärkung der Innovationskraft (Innovieren) erreichen (vgl. Altherr, 2018, S. 412 f.). Dabei werden nicht nur agile Prozesse und Führungswerkzeuge etabliert, sondern vielmehr das generelle Organisations- und Führungsprinzip angepasst (vgl. Rigby et al., 2018). Diese "Adaptionswelle“ deutet darauf hin, dass das „Agilitätsmanagement” - egal in welcher Ausprägung - Erfolge zeigt bzw. zumindest in hohem Maße verspricht.

Die grundsätzliche Eigenschaft, sich agil bezüglich Marktund Technologieveränderungen zu zeigen, war auf den meisten Märkten immer eine Herausforderung. Manchmal braucht es aber eben die richtige Zeit, die ein bestimmtes Verhalten ganz besonders bedeutend macht und/oder einen systematischen Ansatz erfordert. Wie McKinsey (vgl. Aghina et al., 2018) herausarbeiten, gibt es wesentliche 
Abb. 1: Reaktive und proaktive Agilität in Folge äußerer Dynamik

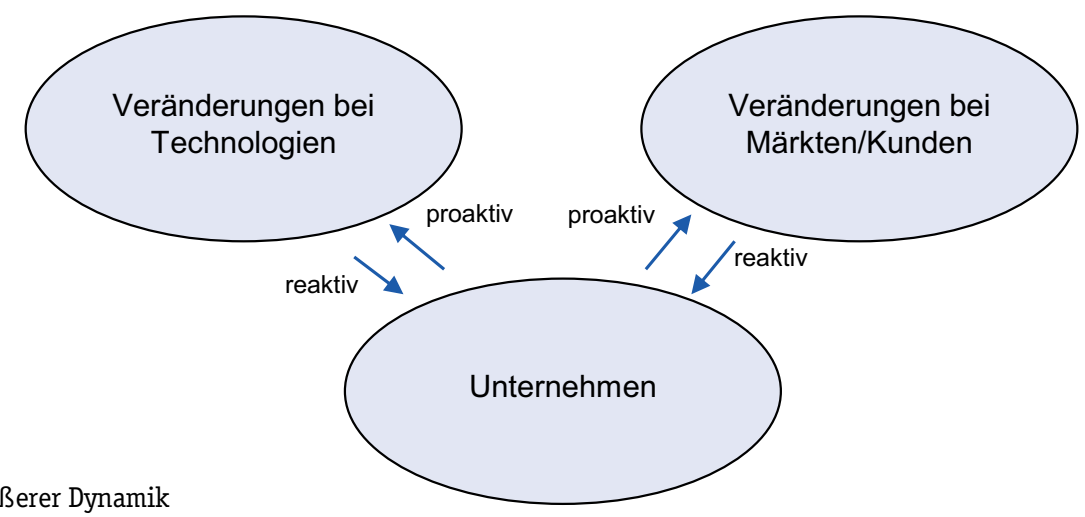

Treiber, die die Notwendigkeit einer umfassenden „agile approach“ und damit den Hype hervorbrachte:

- Sehr häufige disruptive Veränderungen bei Technologien und Märkten

- Ebenfalls sehr schnelle Veränderungen mit neuen Anforderungen bei allen Stakeholdern (Kunden, Investoren, Staat mit neuen Gesetzen) und Wettbewerbern

- Die durchdringende Digitalisierung benötigt ebenfalls 0rganisationen, welche mit multidimensionalen Kommunikationsmustern und komplexen Kollaborationen mithalten können

- Der „Krieg um Talente“ die letztlich andere Ansprüche an Lernen, Arbeit und Verantwortung haben, bedingt zudem die Abkehr von wenig dynamischen Silo- bzw. Senioritätskarrieren

Die - wenn man so will - Notwendigkeit, agile Konzepte in Unternehmen einzuführen, lässt sich ähnlich auch über das VUCA-Modell begründen; VUCA steht dabei als Abkürzung für zentrale Aspekte der skizzierten Veränderungen: $\mathrm{V}=$ Volatilität, U = Unsicherheit, C = Komplexität, A = Ambiguität. Auf jeden Fall scheint es über neuere exogene Entwicklungen genug Anlass zu geben, systematischer reaktiv oder besser noch: Proaktiv an Veränderungen heranzugehen, wie die Abb. 1 zeigt.

\section{Welche Managementaufgaben sind angesprochen?}

Alle klassischen Managementaufgaben (vgl. Bleicher, 1991, S. 450) sind durch Agilität prinzipiell angesprochen. Dies lässt sich wie folgt erläutern: Agilitätsmanagement begreift sich zunächst als die Intention, ein Unternehmen in einer bestimmten Art und Weise zu verändern - nämlich "agil" werden zu lassen. Hierzu sind in den letzten Jahren neue Methoden, organisatorische Muster und Kultur- und Führungsprinzipien entstanden bzw. "alte“ wurden "reaktiviert“. Je nach Managementaspekt (Strategie, Organisation, Führung etc.) werden diese Methoden und organisatorischen Muster entsprechend angewandt bzw. zumindest empfohlen. Bezüglich der einzelnen Elemente des Managements lässt sich folgendes festhalten:
- Zielebene: Vielmehr als in weniger agilen Unternehmen ist es nun notwendig, durch klare Ziele - insbesondere Visionen - für die notwendigen ständigen agilen Schritte (teilweise auch strategischen Kurswechseln) einen starken Rahmen zu geben (vgl. Pal, 2005, S. 14 ff.).

- Strategie: Auch die strategische Planung soll sich nun nicht mehr nur einmal pro Jahr vollziehen und dann wieder festgeschrieben werden. Es gilt die wechselnden Bedürfnisse sowie Meinungen von Kunden und auch anderen Stakeholdern ständig im Auge zu haben und die strategischen Pläne auch entsprechend neu auszurichten. Werkzeuge wie "Customer Journey Maps", die im Buch noch vorgestellt werden, sollen nun auf veränderte Kundenbedürfnisse installiert werden (vgl. Varma, 2015, S. $118 \mathrm{ff}$.).

- Organisation und Prozesse: Diese Elemente des Managements sind ein zentraler Bezugspunkt der agilen Welt. Agilität impliziert flache Hierarchien, passende neue Teamformen und Selbstorganisation. Klassische Funktionalabteilungen spielen eine untergeordnete oder gar keine Rolle mehr.

An einem typischen Beispiel der Organisation der Entwicklung neuer Produkte und Dienstleistungen können verschiedene "Ausbaustufen" unterschieden werden. Abb. 2 zeigt zunächst eine Organisation mit Funktionalbereichen, die einen abteilungsübergreifenden Prozess („Stage Gate Prozess") abwickeln. Wohl gemerkt gibt es in dieser Welt schon abteilungsübergreifende Projektteams. Eine erste agile Ausbaustufe ist dann die Einführung agiler Methoden wie Scrum in dem Stage Gate Prozess, wie es auch inzwischen Cooper vorschlägt ("hybrides“ Stage Gate Modell, vgl. Cooper, 2018, S. 19). Eine nächste Stufe ist die Einrichtung einer wertstromorientierten Organisation, bei der man temporäre Projektteams letztlich dauerhaft für bestimmte Aufgabenstellungen - z.B. zur Entwicklung bestimmter Produktlinien zusammenfasst.

Die Konstellation im Quadranten rechts oben findet man inzwischen bei einigen Unternehmen; Unternehmen wie Spotify haben diese genannten Strukturen bekannt gemacht. Die Expertise der Funktionalabteilungen geht aller- 


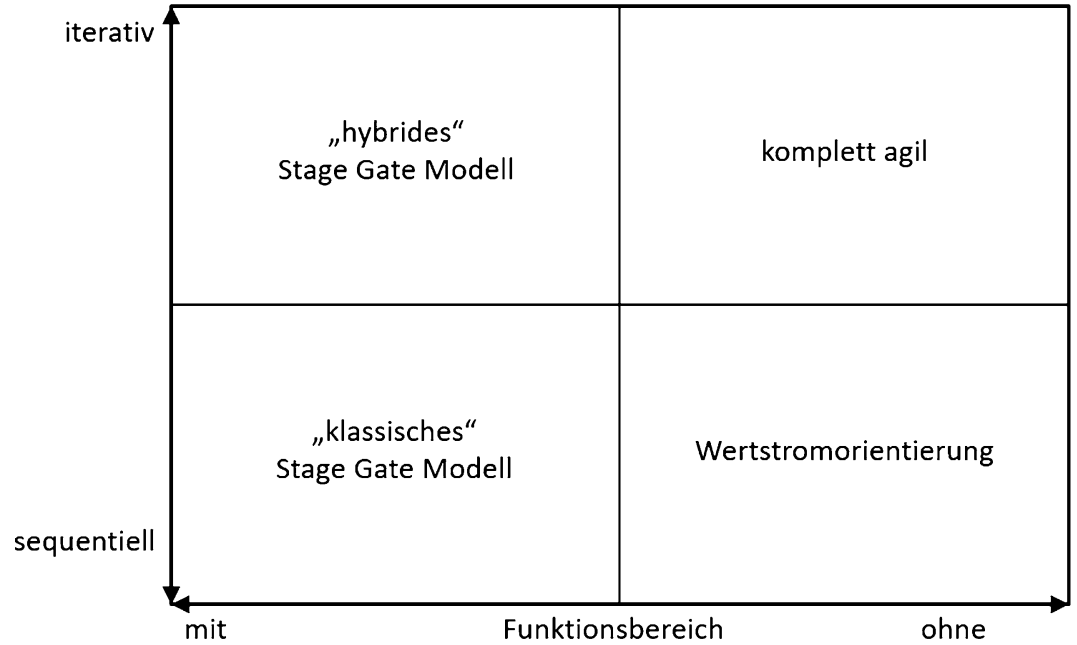

Abb. 2: Mögliche Organisationsformen für die Entwicklung neuer Produkte und Dienstleistungen dings dort oder in ähnlichen Strukturen nicht verloren. Man bildet "Chapters", das sind letztlich Zirkel, innerhalb dieser sich Marketing- oder F\&E- oder Einkaufsspezialisten zusammenfinden.

- Kultur und Führung: Das Leben als agile Person in einem agilen Unternehmen setzt eine entsprechende, auch durch das Management gelebte Kultur voraus. Der inzwischen vielzitierte "Mind Shift" muss vom Management vollzogen und verdeutlicht werden. Zentral ist in puncto Führung das Einräumen von mehr Verantwortung für die Mitarbeiter sowie das wenig direkte Steuern und Kontrollieren. Die Führungskraft versteht sich eher als Coach, die Leitlinien gibt und punktuell Unterstützung anbietet. Somit wird weg vom klassisch hierarchischen Führungsverständnis in agilen Teams viel häufiger ein „Führen ohne formale Macht“ praktiziert. Selbstverständlich kommen auch agile Teams nicht gänzlich ohne Führung aus - die Führungskompetenz ist hierbei jedoch nicht zwangsläufig auf Grundlage von Formalitäten an eine einzelne Person gebunden. Vielmehr beruht die Verteilung der Führungskompetenzen in agilen Teams auf der Akzeptanz durch die Gruppe, welche in den meisten Fällen durch fachliche Expertise und persönliche Eigenschaften (z.B. Charisma, Rhetorik) begründet ist (vgl. Altherr, 2018, S. 422 ff.). In einigen agilen Teams werden demnach die Führungskompetenzen rollenbasiert auf die unterschiedlichen Teammitglieder aufgeteilt. Der sog. Shared-Leadership Ansatz fordert von den Teammitgliedern jedoch auch ein hohes Maß an Rollenmobilität, Adaptivität und Selbstverantwortung (vgl. Döös, 2015; vgl. Martin et al., 2018). Auf der einen Seite ist es von Bedeutung, dass Mitarbeiter weitgehend die Mobilität erhalten, ihre Rollen im Unternehmen zu ändern und entsprechende Erfahrungen zu gewinnen. Auf der anderen Seite ist das nicht durch jeden Mitarbeiter sofort leistbar, weswegen die Einführung von Selbstorganisation mit individuell auf das Unternehmen abgestimmten Entwicklungs- maßnahmen begleitet werden sollte (vgl. Altherr, 2018, S. 425).

- Operative Planung und Kontrolle: Hier gibt es eine Vielzahl von Werkzeugen und Methoden, welche agilere Vorgehensweisen ermöglichen. An dieser Stelle möchten wir nur einen Aspekt herausgreifen: Bei sehr flexiblen Vorgehensweisen, bei denen starre Pläne kaum noch sinnvoll sind, werden entsprechend auch Controllingwerkzeuge benötigt, welche diesen Anforderungen gerecht werden. Auch hier haben sich schon erfolgreiche Praktiken etabliert, die agiles Handeln unterstützen. So z.B. entstanden im Rahmen der Scrum-Methodik Kennzahlen, die den Erfordernissen Schnelligkeit und rasche Anpassungsfähigkeit Rechnung tragen. Bei Scrum werden Wege zu einem a priori nicht exakt zu beschreibenden Ziel in "Sprints" eingeteilt. Indikatoren, wie die Kennzahl "Product Increment" oder sogenannte "Velocity", messen dann letztlich, wie gut und wie schnell man sich gerade auf dem agilen Weg befindet (vgl. Rubin, 2013). Firmen wie Bosch, die in manchen Bereichen bereits auf eine vollständige agile Organisation umgestellt haben, empfehlen Methoden wie Design Thinking, Lean Start up und weitere (siehe Abb. 3) in Relation zur Situationsunsicherheit (z.B. Marktbekanntheit) und Komplexität (z.B. Technologiebekanntheit) (vgl. Friedmann 2019, S. 98 ff.). Dieser Gedanke geht auf Stacey zurück (vgl. Stacey, 2001).

Wie wir nun erläutert haben, tangiert der Anspruch, agil zu sein, tendenziell alle klassischen Aufgabengebiete des Managements. Allerdings stellt sich natürlich die Frage, wann überhaupt ein systematisches Agilitätsmanagement bzw. zumindest ein mehr an Agilität in einzelnen Teilbereichen notwendig ist. Sehr vereinfacht lässt sich die Entscheidung, wann agiles Handeln notwendig bzw. wichtig ist, an folgendem einfachen Schaubild skizzieren.

Allerdings gibt die Abb. 4 keine Anhaltspunkte für die passende inhaltliche Ausgestaltung. Es wird damit noch nicht 
Abb. 3: Methodeneinsatz in Abhängigkeit von Komplexität und Unsicherheit

Abb. 4: Wann ist Agilität sinnvoll?
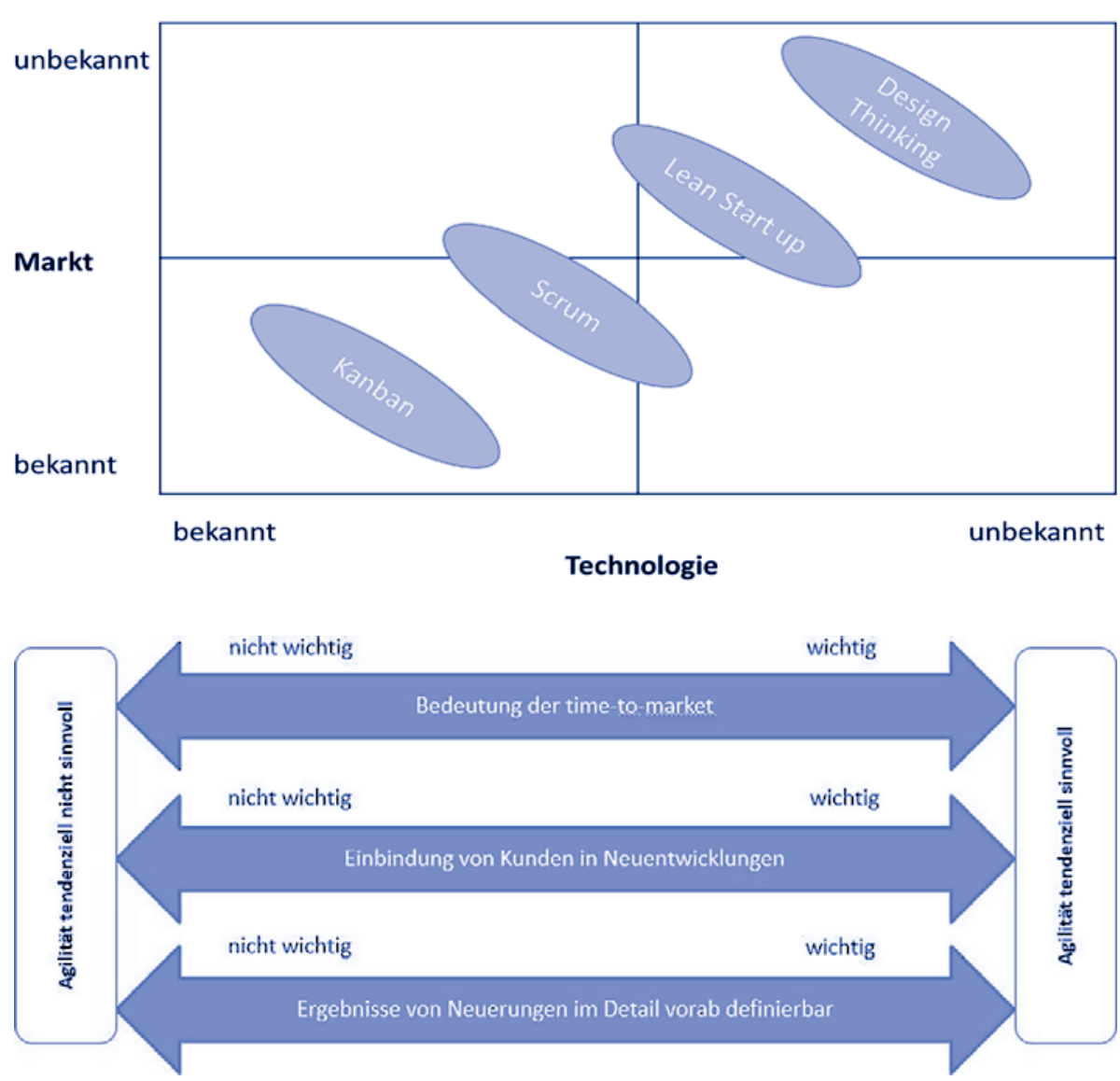

klar, welche Prozesse (operativ oder strategisch), welche Bereiche (F\&E, Vertrieb etc.) oder das gesamte Unternehmen agiler werden müssen. Je nachdem, welche Veränderungsdynamiken in einer Branche zugrunde liegen, werden auch unterschiedliche organisatorische Muster, Methoden etc. benötigt. Es gibt letztlich auch keine digitale Entscheidung "agil“ oder „nicht agil“; auf diese Thematik werden wir im nächsten Abschnitt eingehen

\section{Ambidextrie - oder: Agilität nur dann, wenn sie sich lohnt}

Häufig wird angemahnt, dass eine umfassende Transformation in Richtung Agilität nicht anzuraten sei. Es wird die Notwendigkeit postuliert, bestehende, wenig dynamische Geschäfte "effizient" in klar definierten Prozessen und mit althergebrachten Abteilungen abzuwickeln; neue Geschäfte und/oder auf dynamischen Märkten würden durchaus Agilität verlangen. "Effiziente" und "agile“ Organisationen werden dort in gewisser Weise als konträr erachtet, nichtsdestotrotz sei beides nach Bedarf vorzuhalten bzw. einzusetzen: Ambidextrie, was so viel wie Beidhändigkeit bedeutet, sei gefordert. Auch wenn der Ursprung der Ambidextrieforschung bereits in den 90ern zu finden ist (vgl. Tushman/0'Reilly, 1996), wird das Konstrukt der organisationalen Ambidextrie in aktueller Praxisliteratur verarbeitet (vgl. z.B. Buhse, 2020).
Andere Autoren (Grabmeier, Aghina) sehen den Gegensatz zwischen Effizienz und Agilität weniger. Durchaus sei es wichtig, je nach Situation und Bedarf Agilität zu zeigen, allerdings benötige Agilität letztlich immer stabile Strukturen. Stabile Kernelemente einer Organisation gepaart mit Agilität führen auch zu Effizienz.

Solche Debatten sind u.a. auch Konsequenzen unscharfer Begrifflichkeiten und im Grunde für die Praxis wenig hilfreich. Wie wir bereits erläutert haben, geht es zunächst immer darum zu fragen, wo und wie mehr Agilität sinnvoll ist. Ob und in welcher Form agile Methoden, Organisationsmuster u.ä. gewählt werden, ist dann eine Frage von Aufwand und Ertrag. Wenn z.B. die Einführung eines agilen Organisationsmodells und eine Scrum-Methodik hilft, die Time-to-Market der Entwicklung so signifikant zu verbessern, dass der Aufwand der Transformation klar durch den Ertrag übertroffen wird, dann lohnt eben diese Veränderung.

Als Kern der Ambidextriedebatte lassen sich schlicht zwei Feststellungen treffen:

- Ohne Zweifel ist eine komplette „Agilisierung“ eines Unternehmens in vielen Fällen nicht sinnvoll bzw. notwendig. Wenn z.B. die Ergebnisse unternehmerischer Aufgaben - Produkte und Dienstleistungen - klar definiert sind und ebenso die operativen Prozesse dazu, dann bieten sequentielle Abläufe auch in hierarchischen Strukturen wohl nach wie vor die effizientere 0rganisationsform. 
- Welche agilen Organisationsformen, Führungsprinzipien oder Methoden wann wie Sinn machen, muss im Zweifel untersucht werden. Der Nettowert der agilen Transformation muss schlichtweg positiv sein. Wie man verfahren könnte, um für den jeweiligen Zweck die passende Form der agilen Transformation zu wählen, zeigen Thomaschewski und Völker im Band Praxiswissen Management (vgl. Thomaschewski/Völker, 2019, S. 25 f.)

Als Vorteil der Ambidextriedebatte lässt sich festhalten, dass sie Unternehmen darauf hinweist, das Wann und Wo agiler Konzepte zu hinterfragen. In diesem Kontext wird die Debatte weitens dazu führen, dass Praxis und Wissenschaft noch genauer ausloten müssen, wie das Konzept Agilität weiter konkretisiert werden kann.

\section{Fazit}

Agiles Verhalten ist - so haben wir argumentiert - im zunehmenden Zeichen der Digitalisierung und entsprechenden marktlichen und technologischen Veränderungen eine zentrale Herausforderung für wohl die meisten Unternehmen. Insofern ist es naheliegend, dass das „Management der Agilität“ im Fokus der Managementliteratur und -praxis steht. Wie andere Managementkonzepte durchläuft dieser Ansatz einen Hype Cycle. Wie ebenfalls bei vielen solcher Ansätze findet sich z.T. Bekanntes gepaart mit neuen Einsichten oder neuen oder zumindest für den neuen Zweck angepassten Methoden zu Verhaltensmustern. Agilitätsmanagement tangiert letztlich alle Elemente des Managements; Ziele, Strategien und operative Planung, Kontrolle, Organisation und Führung sind angesprochen. Eine agile Transformation ist aufwendig und teuer; eventuell ist sie auch meist für jede Branche bzw. jedes Unternehmen in einer Branche nicht gleich wichtig. Es gibt zudem auch Aufgaben innerhalb eines Unternehmens, bei denen agiles Verhalten eher untergeordnet oder weniger dringlich ist. Es gilt letztlich für die Ambidextrieanforderungen gewappnet zu sein.

\section{Literatur}

Aghina, W. et al., The five trademarks of agile organizations, in: McKinsey Report, online im Internet: URL: https://www.mckinsey.com/business-fu nctions/organization/our-insights/the-five-trademarks-of-agile-organiz ations\#0, Abrufdatum (05.05.2020).

Altherr, M., Die Organisation der Selbstorganisation, in: Kels, P., KaudelaBaum, S. (Hrsg.), Experten führen, Wiesbaden 2018, S. 411-426.

Beck, K. et al., Principles behind the Agile Manifesto, Snowbird (USA), online im Internet: URL: http://agilemanifesto.org/prinziples.html, (Abrufdatum: 08.05.2020).

Buhse, W., Ambidextrie wird zum Führungsmodell, online im Internet, URL: https://www.cio.de/a/ambidextrie-wird-zum-fuehrungsmodell,358 0207, (Abrufdatum: 27.03.2020).

Bleicher, K., Das Konzept integriertes Management, Frankfurt 1991.

Cooper, R./Sommer A.-F., Agile-Stage-Gate for Manufacturers - Changing the Way New Products Are Developed, in: Research-Technology Management, Vol. 61 (2018), S. 17-26.

Döös, M., Together as One: Shared Leadership Between Managers, in: International Journal of Business and Management, Vol. 10 (2015), S. 45-58. Friedmann, D., Agile Zusammenarbeit zwischen Teams und Führungskräften, in: Thomaschewski, D., Völker, $R$.

(Hrsg.), Agiles Management, Stuttgart 2019, S. 97-114.

Grambeier, S., Ambidextrie als Organisationsprinzip - Innovationen und Kerngeschäft verbinden, online im Internet: URL: https://grabmeier.kien baum.com/2018/03/03/ambidextrie-als-organisationsprinzip-innovatio nen-und-kerngeschaeft-verbinden/(Abrufdatum: 05.05.2020).

Kalenda, M. et al., Scaling agile in large organizations: Practices, challenges, and success factors, in: Journal of Software - Evolution and Process, Vol. 30 (2018).

Martin, J. et al., Shared leadership and team performance: An analysis of moderating factors, in: Procedia Computer Science, Vol. 138 (2018), S. 671-679.

Moss-Kanter, R., Change masters: Innovation and entrepreneurship in the American corporation, New York 1985.

Pal, N./Lim, M., Emergence of the Agile Enterprise, in: Pal, N., Pantaleo, D. (Hrsg.) The agile Enterprise, New York 2005, S. 11-31.

Peters, T./Austin, N., Leistung aus Leidenschaft. Über Management und Führung, Hamburg 1986.

Rubin, K., Essential SCRUM: A Practical Guide to the most popular Agile Process, New Jersey 2013.

Rigby, D.K. et al., Agile at Scale, in: Harvard Business Review, 2018, No. 5/6, S. 88-96.

Stacey, R., Complexity and the Group Matrix, in: Group Analysis, Vol. 34 (2001), S. 221-239.

Thomaschewski D./Völker, R., Agilität und Agilitätsmanagement - eine Einführung, in: Thomaschewski, D., Völker, $R$.

(Hrsg.), Agiles Management, Stuttgart 2019, S. 15-27.

Tushman, M. L./O'Reilly, C. A, Ambidextrous Organizations: Managing Evolutionary and Revolutionary Change, in: California Management Review, Vol. 4 (1996), S. 8-30.

Varma, T., Agile Product Development - How to design innovative products that create customer value, New York 2015. 